http://jmscr.igmpublication.org/home/ ISSN (e)-2347-176x ISSN (p) 2455-0450 crossref DOI: https://dx.doi.org/10.18535/jmscr/v10i1.04

\title{
Significance of Frozen Section and Squash Cytology in Diagnosing C.N.S. Lesions and It's Histopathological Correlation
}

\author{
Authors \\ Ambrish Kumar', Jini L Valooran ${ }^{2}$ \\ ${ }^{1}$ Assistant Professor, Department of Pathology, K D Medical College Mathura, Uttar Pradesh, India \\ ${ }^{2}$ Associate Professor, Department of Pathology, Govt. Medical College, Thrissur, Kerala, India \\ Corresponding Author
}

Khethmal P

\begin{abstract}
Introduction: Intraoperative evaluation of lesions in central nervous system requires the correlation of clinical, radiological and histologic data and knowledge of clinicopathological entities and their common locations. The rapid diagnostic evaluation at Frozen Section may guide intraoperative management, which may be particularly important during open craniotomy. In addition, Frozen section allows for the appropriate triage of tissue for ancillary studies such as electron microscopy, microbiologic cultures, and frozen tissue storage. Intraoperatve consultations have become increasingly common in suspected cases of central nervous system. neoplasms. The study is designed to assess the accuracy of squash cytology and frozen section in diagnosing the CNS lesions on intra-operative biopsies in comparison to the gold standard paraffin embedded histopathological sections report of post operative specimens.

Methods: Diagnostic test evaluation. All the cases received from neurosurgery department for intraoperative diagnosis.

Results: Frozen section shows significant correlation with histopathology in CNS lesions

Conclusions: The role of Frozen Section in the intraoperative consultation is important. Our results show a high percentage of accuracy in the intraoperative diagnosis of CNS lesions.

Keywords: FS-Frozen section, SC-Squash cytology.
\end{abstract}

\section{Introduction}

Primary CNS tumours are relatively infrequent in comparison with other malignant tumours. ${ }^{[1,2]}$ It is estimated that the annual incidence of $\mathrm{CNS}$ tumours ranges from $10-17$ per 1,00,000 persons for intracranial tumour and 1 to 2 per 1,00,000 persons for intraspinal tumours. Neuroepithelial tumours are proportionally more frequent in children. ${ }^{[3]}$ Intraoperative evaluation of lesions in central nervous system requires the correlation of clinical, radiological and histological data and knowledge of clinico-pathological entities and their common locations. Advances in the neuroimaging during the last 20 years have revolutionized the diagnosis and treatment of C.N.S. diseases. The diagnosis and treatment of patients have improved because of these changes and have allowed access to regions that were previously inaccessible. These new approaches have placed pathologists in a key role in diagnosis of C.N.S. lesions, especially tumours. The rapid diagnostic evaluation at FS (Frozen Section) may 
guide intraoperative management, which may be particularly important during open craniotomy surgeries. ${ }^{[4,5]}$ In addition, FS allows for the appropriate triage of tissue for ancillary studies such as electron microscopy, microbiologic cultures, and frozen tissue storage. Intraoperatve consultations have become increasingly common in suspected cases of central nervous system neoplasms. The major criteria for requesting an intraoperative diagnosis may vary

(a) If intraoperative management will be influenced

(b) If an unexpected lesion seen at surgery different from what was expected clinically

(c) If main aim is to obtain a biopsy diagnosis

(d) To assess margins, if radical excision is planned

In some centres, neuropathologists rely on cytology smears prepared by squash method, while in others both SC (Squash cytology) and FS are employed. Generally soft consistency of most primary tumours of central nervous system facilitates preparation of squash smears and SC is being used since long for intra-operative diagnosis with a great degree of success. FS are mainly useful for the more firm, rubbery neoplasms such as meningiomas, ependymomas and most metastatic tumours and again gives almost a clear picture of the neoplasms. Several studies suggest that a combination of the two (SC and FS) techniques is most beneficial and gives most of the time accurate diagnosis. The specimen obtained in a craniotomy, for example, will likely allow for the use of cytologic squash techniques coupled with frozen section and can usually rather most often provides a specific and definitive diagnosis. If a high-grade glial neoplasm is present, the treatment may be impacted and adjuvant chemotherapy is instituted at the time of surgical procedure. However, the very small samples obtained by stereotactic biopsies may limit the intra-operative interpration to that of "lesional or diagnostic tissue obtained" with a further deferral to permanent sections. In these cases, it is utmost importance that the neurosurgeon is aware of that diagnostic material has been sampled. Intra-operative consultation of C.N.S. lesions provides accurate diagnosis to neurosurgeons. Some lesions, however may cause diagnostic difficulty. In this study accuracy of intra-operative consultations of central nervous system lesions and discrepancies in diagnosis and deferrals will be analysed. The study is designed to assess the accuracy of squash cytology and frozen section in diagnosing the CNS lesions on intra-operative biopsies in comparison to the gold standard paraffin embedded histopathological sections report of post operative specimens.

\section{Materials and Methods}

Study design is diagnostic test evaluation. All the cases received from neurosurgery department for intraoperative diagnosis in Govt Medical College Thrissur during study period of 18 month. All biopsy material received was submitted for the squash and frozen section in which a tiny part $(1 \mathrm{~mm})$ of tissue was taken over slide and smeared. 2 such smears were prepared, one of which was immediately stained with methylene blue and examined and other smear was fixed in isopropanolol $(85 \%)$ and rapid pap-stain was done. Both the smears was examined under microscope and the suggestive diagnosis was made correlating with the site of the lesion, age and sex of the patient and the clinical as well as radiological findings.

Other part of the biopsied tissue bits was processed in Leica CM 1850 Cryostat microtome and 2 frozen section slides were prepared and each slide was fixed in the iso-propanolol $(85 \%)$ and stained by rapid Haematoxylin and Eosin method and were studied under microscope.

The diagnosis thus made on the basis of squash and frozen section was conveyed to the neurosurgeons within $40 \mathrm{~min}$, the standard reporting time for the intra-operative diagnosis by frozen sections.

All the diagnoses were compared with the diagnoses made on post-operative specimen, 
routine paraffin embedded sections of histopathology, which is the gold standard.

\section{Observations}

In all 83 cases intraoperative squash smear preparation, frozen section, clinical history, radiological diagnosis, cytological findings of methylene blue and pap-stained smears, rapid $H \& E$ stained frozen section findings and final histopathological diagnosis were recorded.

(Table 01) Showing agewise distribution of cases The youngest patient was only 10 months old and was a case of anaplastic ependymoma and the oldest was 82 years old. The maximum number of patients were in the age group of 40-60 years, 57.8 $\%$ (48 cases) followed by 21-40 years, $18.10 \%$ (15 cases)

Table 01 Age wise Distribution

\begin{tabular}{|l|c|c|c|} 
Group & $\begin{array}{c}\text { Age Range in } \\
\text { years }\end{array}$ & Frequency & Percent \\
\hline 1 & $0-20$ & 5 & $6.0 \%$ \\
\hline 2 & $21-40$ & 15 & $18.1 \%$ \\
\hline 3 & $41-60$ & 48 & $57.8 \%$ \\
4 & $61-80$ & 14 & $16.9 \%$ \\
5 & $>80$ & 1 & $1.2 \%$ \\
& Total & 83 & $100 \%$ \\
\hline
\end{tabular}

(Table 02) Showing gender distribution Male: female ratio observed in the study was found to be 0.73: 1 (54.22\% females and $45.78 \%$ males). Several studies have got the similar ratio.

Table 02 Gender Distribution

\begin{tabular}{|c|c|c|} 
Gender & Frequency & Percent \\
Males & 38 & $45.8 \%$ \\
Females & 45 & $54.2 \%$ \\
Total & 83 & $100 \%$ \\
\hline
\end{tabular}

Table 03 Showing case distribution according to site
Table 03 Distribution occording to site

\begin{tabular}{l|cc} 
Various Sites of the Tumours & Frequency & Percent \\
Frontal & 19 & $22.9 \%$ \\
Temporal / Temporoparietal & 14 & $16.9 \%$ \\
\hline Parietal/ Parieto-Occipital & 13 & $15.7 \%$ \\
\hline Suprasellar & 9 & $10.8 \%$ \\
\hline Intraspinal & 12 & $14.5 \%$ \\
Falx & 6 & $7.2 \%$ \\
Cerebellar & 2 & $2.4 \%$ \\
Cp Angle & 8 & $9.6 \%$ \\
\hline Total & 83 & $100 \%$ \\
\hline
\end{tabular}

Table 04 Case Distribution among Various WHO Categories of CNS Tumours

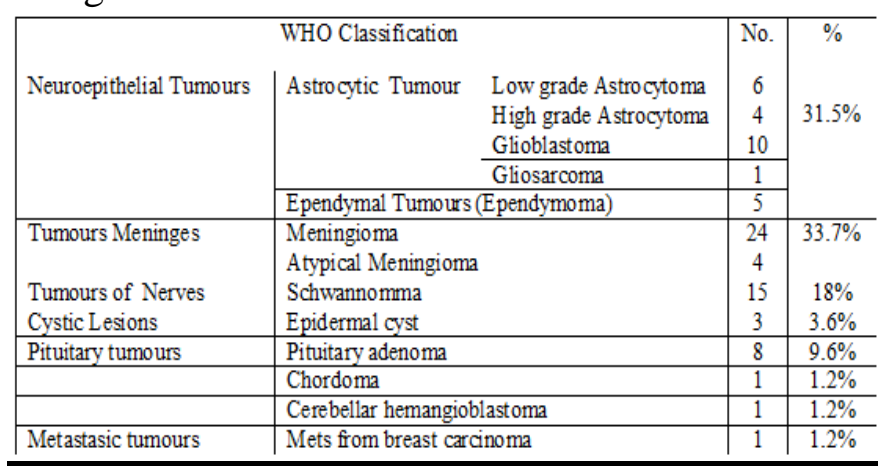

Table 05 Correlation Findings

[FROZEN \& SQUASH]

\begin{tabular}{l|c|c}
\hline Cases & Frequency & Percent \\
Concordant & 80 & $96.4 \%$ \\
Discordant & 3 & $3.6 \%$ \\
Total & 83 & $100 \%$ \\
\hline
\end{tabular}
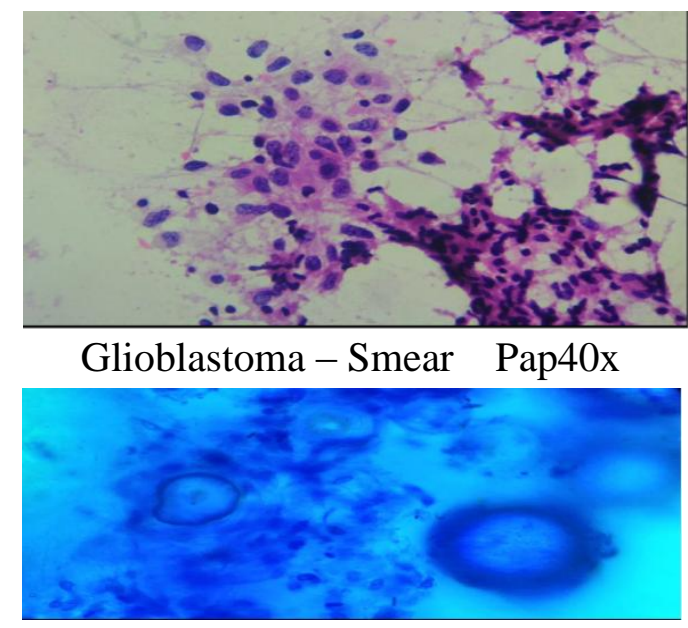

Psammomatous Meningioma-Smear 40x

Table 06 SQUASH Cytology Correlation

\begin{tabular}{|l|cc|}
\hline Cases & Frequency & Percent \\
Concordant & 75 & $90 \%$ \\
Discordant & 8 & $10 \%$ \\
Total & 83 & $100 \%$ \\
\hline
\end{tabular}




\section{Discussion}

Intraoperative consultation (FS and SC) can provide surgeons with a primary diagnosis that is helpful to decide a subsequent surgical approach. It can inform the surgeon as to whether the biopsy is taken from the appropriate area and the adequacy of the specimen that is important to the pathologist to make a final diagnosis on the permanent sections can be determined (2). The reported diagnostic accuracy of CNS Frozen Section diagnosis is between $85-90 \%$ in previous studies while it is greater with a $97 \%$ agreement degree between final histopathological diagnosis with that of the FS and SC.

In present study, nearly $80 \%$ of CNS tumours encountered were between the age of 20 and 60 years which was comparable with Korean study who observed about $70 \%$ in the same age group. Glioblastoma was the common tumour in older patients while ependymomas showed a predilection for younger age group.

Male: Female ratio in the present study was found 0.73:1 which was comparable with Amarati et al (0.75:1), Bharati Jha et al (0.77:1). In the present study $85.29 \%$ tumours were intracranial and $14.21 \%$ were spinal. The Andrews et al and KNP study also found $88.66 \%$ intracranial, $13.34 \%$ spinal and $93.5 \%$ intracranial, $6.5 \%$ spinal respectively. Meningioma was the commonest (33.7\%) tumour encountered in our study. Comparable findings were reported by $Y L$ Suh et al study $^{[6]}$ and CBTRUS study ${ }^{[7]}$. Astrocystic tumours were the 2nd most common tumour observed in the present study (30.6\%). Jerzy et al also reported it as 2nd most common tumour in his study.

All the tumours were completely correlated histologically in the study with an average of $99 \%$ as no discordance was found in categorising lesions as Non-neoplastic / Benign / Malignant lesions. Also there was no discrepancy in correlation in tumour grade as per WHO guidelines, as all the tumours with Low grade / High grade on histopathology were reported same on FS and SC. Cases where SC was misleading,
FS confirmed it as differentiation of low grade astrocytoma and normal cerebral tissue on cytology is difficult sometimes because the astrocytes display minimal to no anaplasia and are loosely dispersed in a delicate fibrillary background and similar experience was experienced in 1 of our case where tissue provided was from the periphery of the lesion and was not representative, a diagnosis of low grade glioma was made on cytology as one the clinical differentials was Glioma. But actual picture was shown by the frozen section on which we found normal brain tisuue with reactive gliosis. Final paraffin embedded sections report was metastasis from carcinoma breast. It was noted that presence of necrosis and marked endothelial proliferation on cytology were in favour of the diagnosis of glioblastoma rather than high-grade astrocytoma as frozen sections clarified the picture.

The majority of discordancies between FS and the permanent diagnoses were seen in ependymoma, glioblastoma, metastatic tumors, oligodendroglioma, meningioma, and astrocytoma $^{[8,9]}$. A French study on1, 315 cases found $96.6 \%$ concordance between FS and permanent diagnoses. Diagnostic accuracy was $92.4 \%$ in Talan Hernilovic ${ }^{[10]}$ and $95 \%$ in Roessler $^{[11]}$. The most accurate FS diagnoses were made in cases of meningioma $(97.9 \%)$, metastasis (96.3\%), and glioblastoma (95.7\%). In a referral center in Pakistan, the diagnostic accuracy was 88.9\% in CNS neoplasms for 171 cases. Our results showed a high accuracy percentage for frozen sections in the diagnosis of CNS lesions (99.5\%), when concordant and partially concordant cases were included.

The majority of discordancies between FS and the HP diagnoses were seen in ependymoma and metastatic tumors ${ }^{[8,9]}$, where as discrepancies were seen more in SC diagnosis where fibrous meningiomas, degenerated schwannomas, reactive gliosis, and low grade astrocytomas [cases were not in HP correlation. Thomas et al had less than $3 \%$ discordancy among 2,156 cases during an 8year period. Approximately $80 \%$ of the 
discrepant cases were spindle cell lesions, astrocytoma versus oligodendroglioma, lymphoma, reactive versus neoplastic process, and tumor overgrading. A French study on 1,315 cases found $96.6 \%$ concordance between FS and permanent diagnoses ${ }^{[10]}$ which is exactly same in the present study. Diagnostic accuracy was $92.4 \%$ in Talan Hernilovic ${ }^{[11]}$ and $95 \%$ in Roessler ${ }^{[12]}$ among 4,172 patients. The most accurate FS diagnoses were made in cases of meningioma (97.9\%), and glioblastoma $(95.7 \%)^{[12]}$. In a referral center in Pakistan, the diagnostic accuracy was $88.9 \%$ in CNS neoplasms for 171 cases. Our results showed a high accuracy percentage for frozen sections in the diagnosis of CNS lesions (99\%), when concordant and partially concordant cases were included and considering partially concordant cases were correct but not as accurate as completely concordant diagnoses. Incorrect FS diagnosis of astrocytomas in some cases could be due to the thickness of the cuts and technical problems with staining which results in the disruption of cellular morphology. Histological diagnosis was made according to the criteria set forth in pathology textbooks for diagnosis of CNS lesions. For histological typing of tumors, WHO recommendations were followed.

\section{Conclusion}

The role of FS in the intraoperative consultation is important. Our results show a high percentage of accuracy in the intraoperative diagnosis of CNS lesions. Appropriate communication between pathologists and neurosurgeons with adequate information about the radiologic findings clinical details and surgical details is helpful to minimize the intraoperative FS and SC misdiagnosis. Also knowledge of the proper techniques of making squash smears, frozen sections, staining and artefacts occurring in FS and SC are equally important while studying the cases.

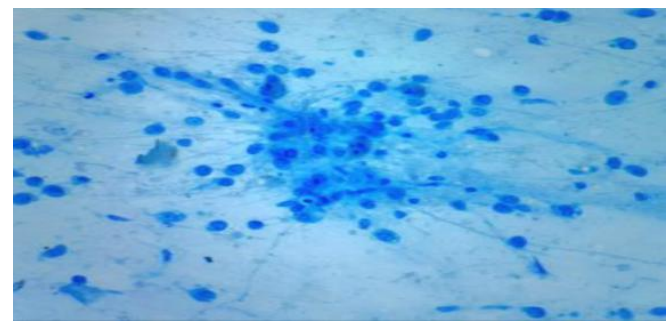

Pilocytic Astrocytoma- smear 40x

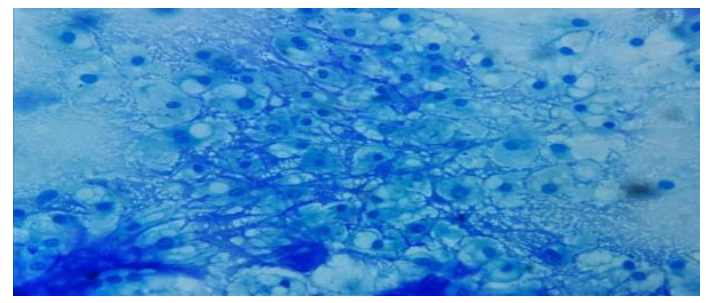

Chordoma-Smear preparation 40x

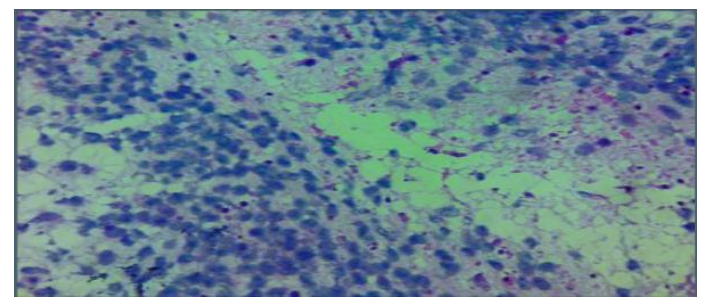

Glioblastoma-Frozen section H\&E 40x

\section{References}

1. Kumar V, Abbas AK, Fausto N, Aster JA. Robbins \& Cotran Pathologic basis of disease 7th ed. Philadeiphia: Elsevier, 2004.

2. Greig NH, Ries LG, Yancik R, Rapoport SI. Increasing annual incidence of primary malignant brain tumours in elderly. J Natl Cancer Inst 1990;82(20):1621-4.

3. Silverberg SG, Delellis RA, Frabe WJ. Principles and practice of Surgical pathology and cytopathology. 3rd edn. New York: Churchil Livingstone. 1997.

4. Yachnis AT. Intraoperative consultation for nervous system lesions.SeminDiagn Pathol.2002;19(4):192-206.

5. Firlik KS, Martinez AJ, Lunsord LD. Use of cytological preparations for the intraoperative diagnosis of stereotactically obtained brain biopsies: a 19 year experience and survey of neuropathologists. J Neurosurg. 1999;91:454-458. 
6. Burger PC, Nelson JS. Stereotactic brain biopsies: specimen preparation and evaluation. Arch Pathol Lab Med. 1997;121:477-480.

7. Folkerth RD. Smears and frozen sections in the intraoperative diagnosis of central nervous system lesions. Neurosurg Clin North Am. 1994;5:1-18.

8. Plesec TP, Prayson RA. Frozen section discrepancy in the evaluation of central nervous system tumors. Arch Pathol Lab Med 2007; 131:1532-40.

9. Savargaonkar P, Farmer PM. Utility of intra-operative consultations for the diagnosis of central nervous system lesions. Ann Clin Lab Sci 2001; 31:133-9.

10. Roessler K, Dietrich W, Kitz K. High diagnostic accuracy of cytologic smears of central nervous system tumors. A 15-year experience based on 4,172 patients. Acta Cytol 2002; 46:667-74.

11. Ud Din N, Memon A, Idress R, Ahmad Z, Hasan S. Central Nervous System Lesions: Correlation of Intraoperative and Final Diagnoses, Six Year Experience at a Referral Centre in a Developing Country, Pakistan. Asian Pac J Cancer Prev 2011; 12:1435-7.

12. Suh YL, Koo H, Kim TS, Chi JG, Park $\mathrm{SH}$, Khang SK, et, al. Tumors of the central nervous system in Korea: a multicenter study of 3221 cases. J

Neurooncol. 2002;56(3):251-9. 